\title{
A BATTLE AGAINST AIDS: NEW PYRAZOLE KEY TO AN OLDER LOCK-REVERSE TRANSCRIPTASE
}

\author{
SONY JACOB K. , SWASTIKA GANGULY \\ Department of Pharmaceutical Sciences and Technology, Birla Institute of Technology, Mesra, Ranchi 835215, Jharkhand, India \\ Email: sonyivin@gmail.com
}

Received: 06 May 2016 Revised and Accepted: 09 Sep 2016

\section{ABSTRACT}

Objective: The reason for the failure of most of the anti-HIV drugs are their poor pharmacokinetics, the poor risk to benefit ratio and the drug resistance. With the objective of developing newer pyrazole scaffolds for effective treatment of HIV, binding mode analysis of designing ligands with the HIV-1RT protein and prediction of key ADME and toxicity parameters of the compounds was in an area of interest.

Methods: In this study, molecular docking studies and ADME-T studies were carried out in designing of some novel pyrazole analogs. The protein (PDB ID: 1RT2) was prepared using the Protein Preparation Wizard (Schrodinger Glide 5.0). ADME parameters calculated by QikProp 3.0v and toxicity of designed analogs checked by using two different online software's namely Lazar and protox.

Results: Most of the designed pyrazole analogs have good oral absorption as well as good binding affinity towards HIV-1 reverse transcriptase.

Conclusion: Finally total 5 analogs (SGS-2, 3, 12, 13 and 14) from the 14 designed leads were found to be best on the basis of molecular docking and ADME-T studies.

Keywords: HIV, Pyrazole analogs, Docking study, ADME-T

(C) 2016 The Authors. Published by Innovare Academic Sciences Pvt Ltd. This is an open access article under the CC BY license (http://creativecommons.org/licenses/by/4. 0/) DOI: http://dx.doi.org/10.22159/ijpps.2016v8i11.12634

\section{INTRODUCTION}

The replication of the retrovirus takes place in two steps. First, the transcription of a single-stranded (+) RNA genome into a doublestranded DNA and in the second step, an integration of these into the host genome $[1,2]$. It is a complex process and requires the concerted function of both the DNA polymerase and the ribonuclease $\mathrm{H}$. Thus, in the replication of HIV-1 reverse transcriptase play a pivotal role and is a primary target for antiviral drug development.

The nonnucleoside reverse transcriptase inhibitors are a category of drugs that have been developed and approved by USFDA for the treatment of HIV-1 infection [2, 3]. The design and development of the first generation HIV-1 nonnucleoside reverse transcriptase inhibitors were failed owing to their inflexibility to interact with their specific binding pockets. Resistance exerted by the wild-type HIV-1 strains also were attributed to this fact. In addition to their high specificity to the binding pockets, high potency, and low cytotoxicity made the second-generation nonnucleoside reverse transcriptase inhibitors the drug of choice to treat the HIV infection. Examples include nevirapine, delavirdine, efavirenz, etravirine, and rilpivirine [4]. The structural flexibility of second-generation nonnucleoside reverse transcriptase inhibitors, etravirine, rilpivirine is to bind to the mutated nonnucleoside binding pocket thereby being more effective compared to older drugs [5]. However, the poor pharmacokinetics, unsatisfactory side effects and the rapid appearance of drug resistance of the these clinically approved antiHIV drugs compelled the medicinal chemist to develop novel nonnucleoside reverse transcriptase inhibitors or modify the existing nonnucleoside reverse transcriptase inhibitors [6-8].

In addition, to the interactions with reverse transcriptase, there are certain features to be fulfilled by the drugs to interact and bind with nonnucleoside reverse transcriptase inhibitor receptors. To describe in brief, the designed analog has a "butterfly" with one "body" hydrophilic center (site A) and two hydrophobic "wings", mostly aryl moieties (wings B and C) $[9,10]$. One of the "wings" of this butterfly is made of the $\pi$-electron-rich moiety (phenyl or allyl substituents) that interacts through $\pi-\pi$ interactions with a hydrophobic pocket formed mainly by the side chains of aromatic amino acids (Tyr181, Tyr188, Phe227, Trp229 and Tyr318). The other wing is normally represented by a heteroaromatic ring bearing on one side a functional group capable of donating and/or accepting hydrogen bonds with the main chain of the Lys101 and Lys103. Finally, on the butterfly body, a hydrophobic portion fulfills a small pocket formed mainly by the side chains of Lys103, Val106 and Val179. Inactivation of enzymes results from the complexation of the nonnucleoside binding pockets. This leads to changes in its own conformation. Thus, different chemical and structural features of the inhibitors and their side-chain flexibility make the bound nonnucleoside binding pockets to undergo different conformation changes. In addition, mutations of a few amino acids also cause a variation of the nonnucleoside binding pocket properties, which can decrease the affinity of most of the inhibitors $[10,11]$.

Pyrazole analogs have shown significant biological activities such as antipyretic, anti-inflammatory, analgesic, antimicrobial [12], anticancer [13], monoamine oxidative inhibitory [14] and various other activities Recent reports revealed that a pyrazole analog lersivirine had been reported as a nonnucleoside reverse transcriptase inhibitor [15]

During the design of a drug, a balance between pharmacokinetics, toxicology, and efficacy of the drugs are a main area of consideration. A recent report shows that just $12 \%$ or fewer drugs are only being reaching into the market. Nowadays, a medicinal chemist can utilize the tools of Insilco design to reduce this failure rate by a proper prior prediction of properties of the drug candidate [16].

Thus, the main objective of the current investigation is to find out the binding mode analysis of designed ligands with the HIV-1 reverse transcriptase protein (PDB ID-1RT2) especially to analyse the amino acids present in the active binding site of reverse transcriptase, the type and number of binding interactions along with prediction of ADME parameters of the designed compounds. Further, the toxicity of designed analogs has also been performed by using two different online software's namely lazar and protox.

\section{MATERIALS AND METHODS}

All computational analysis was performed on a Red Hat 5.0 Linux platform (Red Hook, NJ) running on a Dell Precision workstation 
(Round Rock, TX) with an Intel Core 2 quad processor and 8 GB of RAM.

\section{ADME prediction}

The prediction of ADME (Absorption, Distribution, Metabolism, and Excretion) properties is considered to be a vital role in the build-up of new drug candidates. The ADME properties of the proposed analogs were generated by the Schrodinger's Qik Prop. This provides ranges for comparing a molecule's properties with those of $95 \%$ of known drugs. It also evaluates the suitability of drugs based on Lipinski's rule of five, which is essential to ensure drug like pharmacokinetics profile while using rational drug design. According to Lipinski's rule of five, a molecule is said to be orally active when its molecular weight (MA) $500 \mathrm{~g} / \mathrm{Mol}$, calculated octanol/water partition coefficient (c LogP) 5, hydrogen bond donor $(\mathrm{HBD})<5$, hydrogen bond acceptor $(\mathrm{HBA})<10$ and the number of rotatable bonds $<5$ [17].

\section{Docking study}

\section{Protein structure preparation}

The X-ray crystal structures of HIV-1-RT protein, PDB ID: 1RT2 (fig. 1) were obtained from the protein data bank (Research Collaboratory for Structural Bioinformatics (RCSB) (http://www. rcsb.org/pdb).

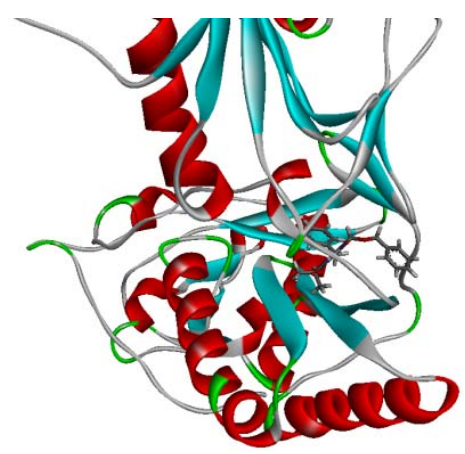

Fig. 1: X-ray crystal structures of HIV-1-RT protein bound with TNK-651

The proteins were then prepared using the Protein Preparation Wizard (Schrodinger Glide 5.0) in which only chain A had been selected for the docking studies. Pre-processed bond orders were assigned, hydrogens were added, metals were treated, and water molecules were deleted. Heterostate for co-crystallized ligand was generated using Epik protonation state and optimization of $\mathrm{H}$ bonding of the protein side-chains were assigned using Prot Assign. The energy was minimized (Impref minimization) using RMSD 0.30 converged by OPLS2005 force field utilities of Schrodinger's Suite 8.5. A radius of $10 \mathrm{~A}{ }^{\circ}$ was selected for active site cavity during receptor grid generation.

\section{Ligand structure preparation}

All the ligands used in the docking study with glide were built within maestro by using build module of Schrodinger Inc. After clean up the structure energy minimization was performed followed by different conformers are generated by maestro tools. Partial atomic charges were computed using the OPLS_2005 force field. Finally, ligprep was prepared for the docking.

\section{Docking protocol and their validation}

All docking calculations were then performed using the "Extra precision" (XP) mode of Glide Program 5.0. A grid was generated with the center defined by the co-crystallized internal ligand of HIV1RT2. During the docking process, initially, Glide performed a complete systematic search of the conformational, orientation and positional space of the docked ligand and eliminated unwanted conformations using scoring followed by energy optimization. Finally, the conformations were further refined via Monte Carlo sampling of pose conformation. The most suitable method of evaluating the accuracy of a docking procedure is to determine how closely the lowest energy poses predicted by the scoring function resembles an experimental binding mode as determined by X-ray crystallography [18]. The reliability of the docking results was checked by comparing the docking scores obtained for the cocrystallized inhibitor with its bound conformation. This was carried out by removing each non-nucleoside reverse transcriptase inhibitor from their active site and redocked into their binding pocket in the conformation found in the crystal structure.

\section{Toxicity studies of designed analogs}

The analogs which have shown a high binding affinity were selected and further subjected to toxicity predictions using two different software's namely lazar, protox software. All of them have different parameters for determining the toxicity of compounds.

\section{Lazar}

Lazar is a modular framework which helps for the prediction of toxic properties based on functional group similarity with mutagenic or carcinogenic parameters by conducting a virtual assay test with Salmonella typhimurium and correlates the results with a standard which are preassigned in the software $[19,20]$. Lazar is freely accessible from http://lazar.insilico.ch all the selected analogs structures (SGS-1 to SGS-14) have been submitted in lazar online web server as input data for predicting the toxicity of the compounds.

\section{Protox}

Computational toxicity studies are having an important role in the reduction of the number of animal experiments, time and cost. Protox is one of the suitable web servers to evaluate the similarity of compounds with known toxic things and toxic fragments. In addition, the web server gives the information about the possible binding affinity of drugs to the different toxicity targets by using various protein-ligand pharmacophore based models [21]. All the selected analogs 2D structures (SGS-1 to SGS-14) have been drawn and submitted for prediction (http://tox. charite. de/tox).

\section{RESULTS AND DISCUSSION}

Reverse transcriptase enzyme which plays a crucial and a multifunctional character in the replication of the human immunodeficiency virus (HIV) and thus exhibits an attractive target for the development of HIV drugs $[18,19]$. Even though, there are a few successful drugs developed the booming prevalence of resistance to these drug candidates and a pulse of their adverse effects made it essential to develop antiviral agents which are active against mutant HIV strains. Thus, in the present investigation, we have designed various pyrazole analogs towards HIV-1 reverse transcriptase they were subjected to molecular docking studies on crystal structures of HIV-1RT2 complexed with ligand TNK-651.

The Qik Prop results of the current investigation have indicated that all the designed molecules are obeying Lipinski rule of five, it's proven its drug-likeness character (table 1). Predicted physicochemical characteristics expressed by various descriptors like the optimum value of rotatable bonds, polar surface area, etc. assures the oral bioavailability of the designed compounds.

The results of intestinal absorption of the designed molecules predicted by Caco-2 cell (QPP Caco) and human serum albumin binding mode predicted by QP log khsa, blood/brain partition coefficient QP $\log \mathrm{BB}$, cell permeability of the blood brain barrier which mimic MDCK cells (QPPMDCK) values also denotes that most of the designed analogs are coming to the prescribed range (table 2). The cell permeability of these analogs also good agreement with their oral absorption rate. Cell permeability, in turn, depends on the partition coefficient and water solubility a compound. The designed analogs (SGS-2,3,6,8,12,13,14) have shown octanol and water (log $\mathrm{Po} / \mathrm{w}<5$ ) and analogs (SGS-2,3,5,7,8,12,13,14) have solubility (QPlogS: -6.5 to 0.5 ) coefficient values in an acceptable range. Further, log BB data shows that all the designed analogs properties in the acceptable range, which indicates drug-like characteristics of designed analogs (table 2). 
Table 1: Lipinski rule of five analyses of designed pyrazole analogs

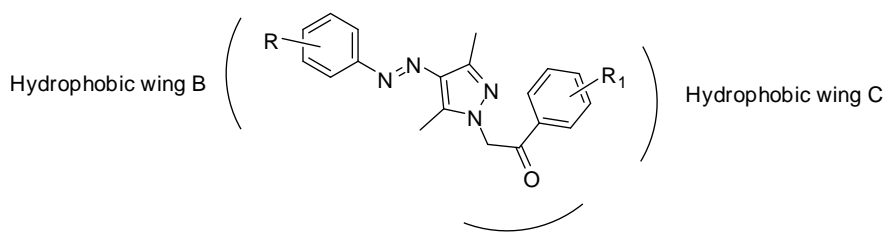

Hydrophillic body Site A

\begin{tabular}{|c|c|c|c|c|c|c|c|c|c|}
\hline Compound code & $\mathbf{R}$ & $\mathbf{R}_{1}$ & $\begin{array}{l}\text { Mol.wt } \\
<500\end{array}$ & $\begin{array}{l}\text { Donor HB } \\
<5\end{array}$ & $\begin{array}{l}\text { Accept } \\
\text { HB } \\
<10\end{array}$ & $\begin{array}{l}\mathrm{QP} \log \mathrm{P}_{0} / \mathrm{w} \\
<5\end{array}$ & $\begin{array}{l}\text { PSA A }^{\circ} \\
7-200\end{array}$ & $\begin{array}{l}\text { \#rotor } \\
<15\end{array}$ & Rule of five \\
\hline SGS-1 & $m-\mathrm{Cl}$ & $\mathrm{Cl}$ & 387.26 & 0 & 5.0 & 5.11 & 62.64 & 5 & 1 \\
\hline SGS-2 & $p-\mathrm{NO}_{2}$ & $\mathrm{Cl}$ & 397.81 & 0 & 6.5 & 3.60 & 97.57 & 6 & 0 \\
\hline SGS-3 & $p-\mathrm{OCH}_{3}$ & $\mathrm{Cl}$ & 382.84 & 0 & 5.75 & 4.66 & 70.94 & 6 & 0 \\
\hline SGS-4 & $3,4 \mathrm{Cl}$ & $\mathrm{Cl}$ & 421.70 & 0 & 5.0 & 5.55 & 62.64 & 5 & 1 \\
\hline SGS-5 & $2,4,5 \mathrm{Cl}$ & $\mathrm{Cl}$ & 456.15 & 0 & 5.0 & 5.9 & 61.47 & 5 & 1 \\
\hline SGS-6 & $2,4-\mathrm{NO}_{2}$ & $\mathrm{Br}$ & 487.26 & 0 & 8.0 & 2.79 & 130.2 & 7 & 0 \\
\hline SGS-7 & $m-\mathrm{Cl}$ & $\mathrm{Br}$ & 431.71 & 0 & 5.0 & 5.23 & 62.64 & 5 & 1 \\
\hline SGS-8 & $p-\mathrm{NO}_{2}$ & $\mathrm{Br}$ & 442.26 & 0 & 6.5 & 3.7 & 97.57 & 6 & 0 \\
\hline SGS-9 & anaphthylamine & $\mathrm{Cl}$ & 402.87 & 0 & 5.0 & 5.59 & 62.62 & 5 & 1 \\
\hline SGS-10 & anaphthylamine & $\mathrm{Br}$ & 447.32 & 0 & 5.0 & 5.70 & 62.62 & 5 & 1 \\
\hline SGS-11 & $3,4 \mathrm{Cl}$ & $\mathrm{Br}$ & 466.15 & 0 & 5.0 & 5.67 & 62.64 & 5 & 1 \\
\hline SGS-12 & $p-\mathrm{OCH}_{3}$ & $\mathrm{Br}$ & 426.06 & 0 & 5.75 & 4.77 & 70.94 & 6 & 0 \\
\hline SGS-13 & $m-\mathrm{NO}_{2}$ & $\mathrm{Cl}$ & 381.82 & 0 & 6.5 & 3.60 & 97.55 & 6 & 0 \\
\hline SGS-14 & $p-\mathrm{OCH}_{3}$ & $\mathrm{CH}_{3}$ & 362.43 & 0 & 5.75 & 4.48 & 70.93 & 6 & 0 \\
\hline
\end{tabular}

Mol. Wt-molecular weight; donor HB-hydrogen bond donor; acceptor HB-hydrogen bond acceptor; QP log P o/w-partition coefficient; PSA A ${ }^{\circ}$-polar surface area; \# rotor-rotatable bonds,rule of five-Number of Lipinsky rule violation

Table 2: ADME-parameters calculated from QikProp

\begin{tabular}{|c|c|c|c|c|c|c|c|c|c|}
\hline $\begin{array}{l}\text { Compound } \\
\text { Code }\end{array}$ & $\mathbf{R}$ & $\mathbf{R}_{1}$ & $\begin{array}{l}\text { 1RT2 } \\
\text { (Kcal/mol) }\end{array}$ & $\begin{array}{l}\text { \% HOA } \\
>80 \% \text { high }<25 \% \\
\text { low }\end{array}$ & $\begin{array}{l}\text { QPP CaCO } \\
<25 \text { poor, } \\
>500 \\
\text { great }\end{array}$ & $\begin{array}{l}\text { QPP Log } \\
\text { BB } \\
-3.0-1.2\end{array}$ & $\begin{array}{l}\text { QP log } \\
\text { khsa } \\
-1.5-1.5\end{array}$ & $\begin{array}{l}\text { QP Log } \\
\text { S } \\
-6.5- \\
0.5\end{array}$ & $\begin{array}{l}\text { QP PMDCK } \\
<25 \text { poor, }>500 \\
\text { great }\end{array}$ \\
\hline SGS-1 & $m-\mathrm{Cl}$ & $\mathrm{Cl}$ & -8.70 & 100 & 2621.6 & -0.03 & 0.52 & -6.41 & 8541.70 \\
\hline SGS-2 & $p-\mathrm{NO}_{2}$ & $\mathrm{Cl}$ & -8.82 & 95.9 & 472.5 & -1.10 & 0.09 & -5.21 & 543.33 \\
\hline SGS-3 & $p-\mathrm{OCH}_{3}$ & $\mathrm{Cl}$ & -8.80 & 100 & 2620.9 & -0.26 & 0.36 & -5.75 & 3461.23 \\
\hline SGS-4 & $3,4 \mathrm{Cl}$ & $\mathrm{Cl}$ & -8.45 & 100 & 2621.6 & 0.09 & 0.63 & -7.05 & 10000.0 \\
\hline SGS-5 & $2,4,5 \mathrm{Cl}$ & $\mathrm{Cl}$ & -8.26 & 100 & 2629.1 & -0.30 & 0.39 & -5.00 & 1365.8 \\
\hline SGS-6 & $2,4-\mathrm{NO}_{2}$ & $\mathrm{Br}$ & -6.95 & 100 & 2559.4 & 0.22 & 0.72 & -7.68 & 10000.0 \\
\hline SGS-7 & $m-\mathrm{Cl}$ & $\mathrm{Br}$ & -7.86 & 100 & 2621.5 & -1.10 & 0.13 & -6.59 & 599.14 \\
\hline SGS-8 & $p-\mathrm{NO}_{2}$ & $\mathrm{Br}$ & -7.53 & 96.5 & 472.5 & -1.10 & 0.13 & -5.38 & 3574.6 \\
\hline SGS-9 & $\begin{array}{l}\text { anaphthyl } \\
\text { amine }\end{array}$ & $\mathrm{Cl}$ & -7.68 & 100 & 2621.5 & -0.22 & 0.79 & -6.96 & 3492.03 \\
\hline SGS-10 & $\begin{array}{l}\text { anaphthyl } \\
\text { amine }\end{array}$ & $\mathrm{Br}$ & -7.89 & 100 & 2621.4 & -0.21 & 0.83 & -6.96 & 3492.03 \\
\hline SGS-11 & $3,4 \mathrm{Cl}$ & $\mathrm{Br}$ & -7.45 & 100 & 2621.6 & 0.11 & 0.67 & -7.23 & 10000.0 \\
\hline SGS-12 & $p-\mathrm{OCH}_{3}$ & $\mathrm{Br}$ & -8.77 & 100 & 2620.8 & -0.25 & 0.40 & -5.92 & 3816.69 \\
\hline SGS-13 & $m-\mathrm{NO}_{2}$ & $\mathrm{Cl}$ & -8.92 & 100 & 474.0 & -1.10 & 0.09 & -5.20 & 545.17 \\
\hline SGS-14 & $p-\mathrm{OCH}_{3}$ & $\mathrm{CH}_{3}$ & -8.72 & 100 & 2621.1 & -0.44 & 0.41 & -5.59 & 1401.82 \\
\hline Standard & TNK-651 & & -13.27 & & & & & & \\
\hline
\end{tabular}

Data indicate the descriptor calculated from Quikprop. Range/ recommended values calculated for 95\% known drugs. \% HOApercentage human oral absorption; QPP Caco-predicted Caco-2 cell permeability; QP log Khsa-predicted blood/brain partition coefficient; QP log Khsa-predicted human serum albumin; QPP MDCK-predicted MDCK permeability; QP log S-predicted aqueous solubility.

The accurate prediction of protein-ligand interaction geometries is essential for the success of virtual screening approaches in structure-based drug design. It requires docking tools that are able to generate suitable conformations of a ligand within a protein binding site and reliable, energetic evaluation indicating the quality of the interaction. The designed pyrazole analogs with the highest docking score (SGS-13) have shown a good binding affinity towards the non-nucleotide binding pocket site of reverse transcriptase enzyme. The dock score of designed analogs and standard drug TNK651 was summarized in table 2 .
To develop more efficient HIV-1 reverse transcription inhibitors, especially active against mutant strains, we further analyzed the various interactions [(hydrogen bonding (backbone and side chain), $\pi-\pi$ interaction of highest docking score analogs towards with amino acids of the binding pocket of HIV-1 reverse transcriptase. Compound SGS-13 had interaction with protein (PDB: 1RT2) through $\pi-\pi$ stacking (Phe 227) and hydrophobic interactions with Val 106, Pro 226, Pro 225,Tyr 318,Leu 234,Trp 229,Tyr 188,Tyr 181, Leu 100, Pro 236 (fig. 2).

The binding pattern of Standard drug TNK 651 also interact with protein (PDB: 1RT2) through $\pi-\pi$ stacking (Trp 229) and hydrophobic interactions (Pro 236, Tyr 181, Pro 95, Pro 225, Val 106, Pro 226, Leu 234, Tyr 318, Leu 100, Val 179, Ile 180, Val 189, Tyr 188 and Phe 227) and hydrogen bond (back bond) interaction with Lys 101 (fig. 3). 


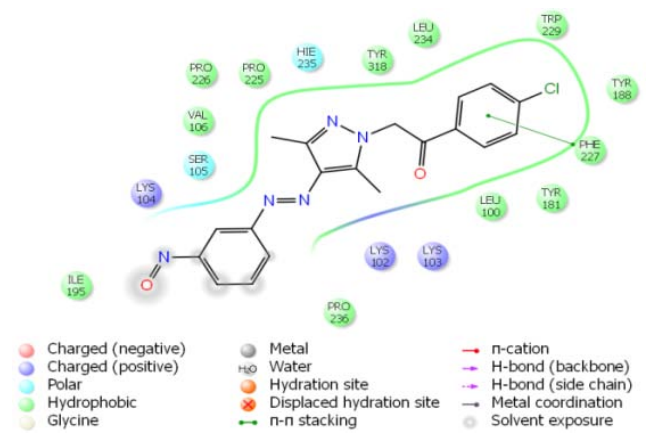

Fig. 2: Ligand interaction pattern of selected analog (SGS-13) into the active site NNBP of 1RT2

The toxicity of selected compounds has been predicted by using lazar and protox. The lazar results have predicted that the designed analogs (SGS-1 to SGS-6, SGS-10, SGS-13) is non-carcinogenic in mouse and rat model. The compound SGS-7 and SGS-8 were predicted to be carcinogenic towards mouse model and rat model, while compounds SGS-9, SGS-11, SGS-12 and SGS-14 has been predicted to be non-carcinogenic towards rat but carcinogenic towards mouse model. The all selected analogs have predicted their mutagenicity towards S. typhimurium. Compound SGS-1 to SGS-7, SGS-11 to SGS-14 was found to be inactive, but compound SGS-8 to SGS 10 has been predicted to be active towards $S$. typhimurium (table 3).

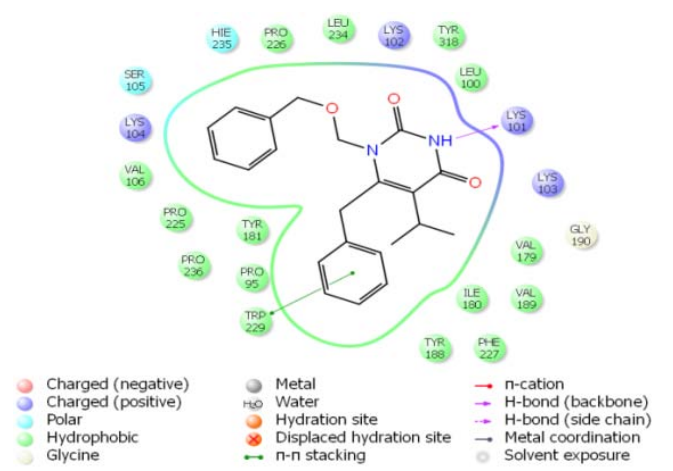

Fig. 3: Redocked conformer of TNK-651 into the active site NNBP of 1RT2

Table 3: Toxicity prediction of selected analogs

\begin{tabular}{|c|c|c|c|c|c|}
\hline Compound code & $\begin{array}{l}\text { Mutagenicity (lazar) } \\
\text { (S. typhimurium) }\end{array}$ & $\begin{array}{l}\text { Carcino } \\
\text { Rat Mou }\end{array}$ & (lazar) & $\begin{array}{l}\text { Protox } \\
\text { predicted } \mathrm{LD}_{50}\end{array}$ & $\begin{array}{l}\text { Protox } \\
\text { predicted toxicity class }\end{array}$ \\
\hline SGS-1 & inactive & inactive & inactive & $629 \mathrm{mg} / \mathrm{kg}$ & CLASS 4 \\
\hline SGS-2 & inactive & inactive & inactive & $5000 \mathrm{mg} / \mathrm{kg}$ & CLASS 5 \\
\hline SGS-3 & inactive & inactive & inactive & $1000 \mathrm{mg} / \mathrm{kg}$ & CLASS 4 \\
\hline SGS-4 & inactive & inactive & inactive & $1000 \mathrm{mg} / \mathrm{kg}$ & CLASS 4 \\
\hline SGS-5 & inactive & inactive & inactive & $629 \mathrm{mg} / \mathrm{kg}$ & CLASS 4 \\
\hline SGS-6 & inactive & inactive & inactive & $629 \mathrm{mg} / \mathrm{kg}$ & CLASS 4 \\
\hline SGS-7 & inactive & active & active & $629 \mathrm{mg} / \mathrm{kg}$ & CLASS 4 \\
\hline SGS-8 & active & active & active & $629 \mathrm{mg} / \mathrm{kg}$ & CLASS 4 \\
\hline SGS-9 & active & inactive & active & $629 \mathrm{mg} / \mathrm{kg}$ & CLASS 4 \\
\hline SGS-10 & active & inactive & inactive & $629 \mathrm{mg} / \mathrm{kg}$ & CLASS 4 \\
\hline SGS-11 & inactive & inactive & active & $629 \mathrm{mg} / \mathrm{kg}$ & CLASS 4 \\
\hline SGS-12 & inactive & inactive & active & $629 \mathrm{mg} / \mathrm{kg}$ & CLASS 4 \\
\hline SGS-13 & inactive & inactive & inactive & $629 \mathrm{mg} / \mathrm{kg}$ & CLASS 4 \\
\hline SGS-14 & inactive & inactive & active & $1000 \mathrm{mg} / \mathrm{kg}$ & CLASS 4 \\
\hline
\end{tabular}

$\mathrm{LD}_{50}$ is defined as the dose at which $50 \%$ of the tested animal die. $\mathrm{LD}_{50}$ data is helping to determine the effective dose of a compound and gives the level of compound's acute toxicity. The acute toxicity data predicted by protox showed that all the selected compounds had high $\mathrm{LD}_{50}$ values that falls under the toxicity class 4 (SGS-1, SGS3 to SGS-14) and toxicity class 5 (SGS-2) according to the GHS, United Nations guidelines (UN GHS, 2005) [20]. From this findings, it was revealed that in future, these designed pyrazole analogs may exhibit better effective inhibition of HIV-1 wild type the most drugresistant mutant strains.

\section{CONCLUSION}

A good binding affinity towards HIV-1 reverse transcriptase and a better predicted oral absorption pattern were observed in the case of all the designed pyrazole analogs. To highlight, a total of 5 analogs (SGS-2, 3, 12, 13 and 14) from the 14 designed leads, were found to be best, on the basis of their molecular docking and ADME-T studies. These analogs may be effective in the inhibition of HIV-1 reverse transcripters strains. Thus, these pyrazole compounds can be made into unique novel anti-HIV drugs.

\section{ACKNOWLEDGMENT}

The authors acknowledge the University Grants Commission (UGC) for financial support in the form of Maulana Azad Fellowship (MANF-2012-13-CHR-KER-13883) and Computer-aided drug design lab, Department of Pharmaceutical Sciences and Technology, Birla Institute of Technology, Mesra for providing necessary facilities to carry out this study.

\section{CONFLICT OF INTERESTS}

The authors confirm that this article content has no conflict of interest

\section{REFERENCES}

1. Hindmarsh P, Leis J. Retroviral DNA integration. Microbiol Mol Biol Rev 1999;63:836-43.

2. Ganguly S, Murugesan S, Prasanthi N, Alptürk O, Herman B, Sluis-Cremer N. Synthesis and anti-HIV-1 activity of a novel series of aminoimidazole analogs. Lett Drug Des Discovery 2010;7:318-23.

3. Himmel DM, Sarafianos SG, Dharmasena S, Hossain MM, McCoy-Simandle K, Ilina T, et al. HIV-1 reverse transcriptase structure with RNase $\mathrm{H}$ inhibitor dihydroxy benzoyl naphthyl hydrazone bound at a novel site. ACS Chem Biol 2006;1:702-12.

4. Kohlstaedt LA, Wang J, Friedman J, Rice P, Steitz T. Crystal structure at 3.5 resolution of HIV-1 reverse transcriptase complexed with an inhibitor. Science 1992;256:1783-90.

5. Sarafianos SG, Das K, Hughes SH, Arnold E. Taking aim at a moving target: designing drugs to inhibit drug-resistant HIV-1 reverse transcriptases. Curr Opin Struct Biol 2004;14:716-30.

6. Martins S, Ramos MJ, Fernandes PA. The current status of the NNRTI family of antiretrovirals used in the HAART regimen against HIV infection. Curr Med Chem 2008;15:1083-95.

7. Sweeney ZK, Klumpp K. Improving non-nucleoside reverse transcriptase Inhibitors for the first-line treatment of HIV infection: the development pipeline and recent clinical data. Curr Opin Drug Discovery Dev 2008;11:458-70. 
8. Daar ES. Emerging resistance pfirtes of newly approved antiretroviral drugs. Topics HIV Med 2007;16:110-6.

9. Sriram D, Bal TR, Yogeeswari P. Newer aminopyrimidine imino isatin analogues as non-nucleoside HIV-1 reverse transcriptase inhibitors for HIV and other opportunistic infections of AIDS: design, synthesis and biological evaluation. Il Farmaco 2005; 60:377-84.

10. Schaefer W, Friebe WG, Leinert H, Mertens A, Poll T, Von der Saal W, et al. Non-nucleoside inhibitors of HIV-1 reverse transcriptase: molecular modeling and X-ray structure investigations. J Med Chem 1993;36:726-32.

11. Ragno R, Frasca S, Manetti F, Brizzi A, Massa S. HIV-reverse transcriptase inhibition: inclusion of ligand-induced fit by cross-docking studies. J Med Chem 2005;48:200-12.

12. Rostom SAF, El-Ashmawy IM, Abd El Razik HA, Badr MH, Ashour HMA. Design and synthesis of some thiazolyl and thiadiazolyl derivatives of antipyrine as a potential non-acidic anti-inflammatory analgesic and antimicrobial agents. Bioorg Med Chem 2009;17:882-95.

13. Zhang D, Wang G, Zhao G, Xu W, Huo L. Synthesis and cytotoxic activity of novel 3-(1H-indol-3-yl)-1H-pyrazole-5-carbohydrazide derivatives. Eur J Med Chem 2011;46:5868-77.

14. Kumar A, Jain S, Parle M, Jain N, Kumar P. 3-aryl-1-phenyl-1hpyrazole derivatives as new multitarget directed ligands for the treatment of Alzheimer's disease with acetylcholinesterase and Monoamine oxidase inhibitory properties. EXCLI J 2013; $12: 1030-42$.
15. Mowbray CE, Burt C, Corbau R, Gayton S, Hawes M, Perros M, et al. Pyrazole NNRTIs 4:Selection of UK-453 061 (lersivirine) as a development candidate. Bioorg Med Chem Lett 2009;19:5857-60.

16. Biopharmaceutical research Industry. Available from: http:// www.phrma.org/sites/default/files/pdf/2015_phrma_profile.p df. [Last accessed 15 Dec 2015].

17. Lipinski CA. Lead-and drug-like compounds: the rule-of-five revolution. Drug Discovery Today Technol 2004;1:337-41.

18. Swastika G, Geeta Y. Molecular docking studies of novel benzimidazole analogs as HIV-1-RT inhibitors with broadspectrum chemotherapeutic properties. Int J Drug Des Discovery 2013;4:1193-13.

19. Maunz A, Gütlein M, Rautenberg M, Vorgrimmler D, Gebele D, Helma C. Lazar: a modular predictive toxicology framework. Front Pharmacol 2013;4:1-0.

20. Helma C. Lazy structure-activity relationships (lazar) for the prediction of rodent carcinogenicity and Salmonella mutagenicity. Mol Diversity 2006;10:147-58.

21. Drwal MN, Banerjee P, Dunkel M, Wettig MR, Preissner R. ProTox: a web server for the in silico prediction of oral rodent toxicity. Nucleic Acids Res 2014;42:W53-8.

\section{How to cite this article}

- $\quad$ Sony Jacob K, Swastika Ganguly. A battle against aids: new pyrazole key to an older lock-reverse transcriptase. Int J Pharm Pharm Sci 2016;8(11):75-79. 